\title{
Numerical Study of Keyhole Centroid Infrared Image Based on Fixed Threshold Method during High Power Fiber Laser Welding
}

\author{
Yu-Quan Chen ${ }^{\mathrm{a}}$, Xiang-Dong Gao ${ }^{\mathrm{a}, *}$, Jian-Yuan Huang ${ }^{\mathrm{a}}$ and Qian Wen ${ }^{\mathrm{a}}$ \\ School of Electromechanical Engineering, Guangdong University of Technology, Guangzhou \\ 510006, China \\ agaoxd666@126.com *Corresponding author
}

Keywords: Molten pool, Seam gap, High-power fiber laser welding, Weld seam.

\begin{abstract}
Seam tracking is significant to obtain good welding quality. Aiming at establishing a model to detect and calculate the seam tracking offset during high-power fiber laser welding of Type 304 austenitic stainless steel plate butt joint welding, an infrared sensitive high-speed camera arranged off-axis orientation of laser beam was applied to capture the dynamic thermal images of molten pools. Through analyzing the molten pool infrared images, the keyhole was extracted by using the fixed threshold method. Extracted keyhole image from different threshold values 0.55, 0.65, 0.85, and 0.93 , were further analyzed to study their differences. Using keyhole images and calculating the keyhole centroid during high-power fiber laser welding, the deviations between the keyhole centroid and the welding seam was analyzed. The welding experiments confirmed that the keyhole centroid was related to the seam offset and the proposed welding seam equation was effective.
\end{abstract}

\section{Introduction}

In industrial welding circumstance, seam tracking is prerequisite to obtain good welding quality [1]. Owing to the high-intensity power and rapid welding speed of high-power fiber laser welding, it is difficult to detect the seam position accurately, especially when the seam gap is less than $0.1 \mathrm{~mm}$. Nowadays more and more visual sensors have been applied in welding process [2]. To detect the very narrow seam position, a promising approach is to identify the seam tracking offset between the laser beam and the welding seam from a molten pool image captured by a camera. But because of the strong spatter and plume influence in the high-power laser welding process, it is difficult to directly obtain a clearer weld image. This is not conducive to judge weld seam position and restricted application and popularization of visual sensing method. Laser welding is a complicated thermodynamic and physicochemical process, which involves material melting, evaporating, plasma forming, keyhole occurrence, and so on. The weld shape can be determined by many parameters in the thermodynamic and physicochemical process, such as the relative speed between the laser and the workpiece. If an electro-mechanical or purely mechanical system is used to drive the welding workpiece, nonlinear factors will also be inevitably introduced to the welding process and influence the system performance [3].

In this paper, through analyzing the infrared image of a molten pool, the keyhole image was extracted by using the fixed threshold method. Extracted keyhole image from different threshold values $0.55,0.65,0.85$, and 0.93 , were further analyzed their differences. Using keyhole images and calculating the keyhole centroid during high power fiber laser welding, the deviations between the keyhole centroid and the welding seam was analyzed. The welding experiments confirmed that the keyhole centroid was related to the seam offset and proposed welding seam equation was effective.

\section{Experiment}

Experimental conditions. The schematic experimental setup for butt joint laser welding is shown in Fig.1. The top side of a welded specimen is shown in Fig.2. In order to research the infrared radiation characteristics of the keyhole related to the seam offset between laser beam focus and welding seam, the laser beam focus was controlled to move along an oblique line across two stainless 
plates. An infrared sensitive high-speed camera captured 2500 frame thermal images of molten pools corresponding to the welding seam of specimen. A continuous wave (CW) fiber laser (IPG YLR-10000) was used for butt joint welding [3], and the welding conditions are listed in Table 1.

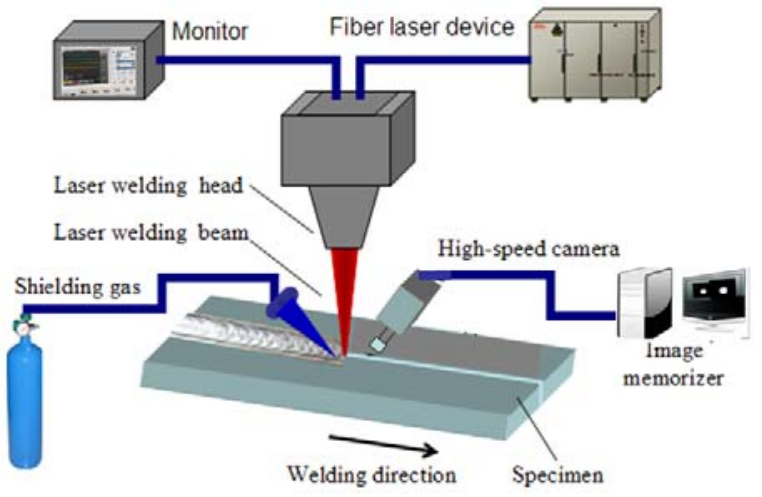

Fig.1 Experimental setup of fiber laser welding

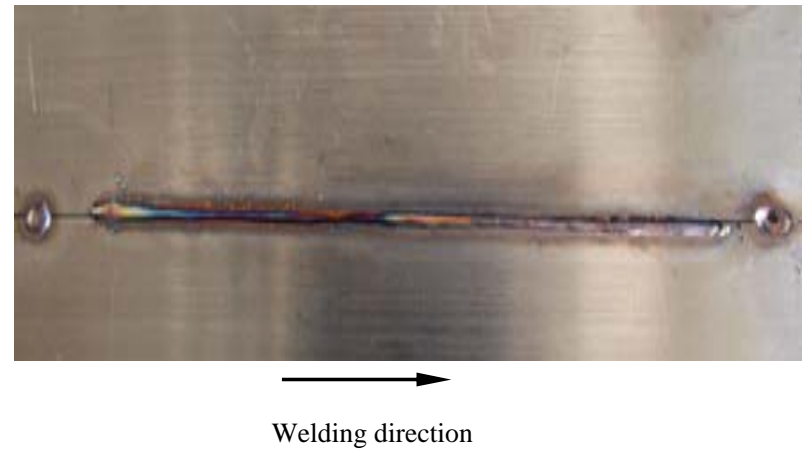

Fig.2 Surface of welded specimen

Table 1 Fiber laser welding conditions

\begin{tabular}{llll}
\hline Laser power & $10 \mathrm{~kW}$ & Filter wave length $\lambda$ & $960-990 \mathrm{~nm}$ \\
\hline Beam diameter & $200 \mu \mathrm{m}$ & Image resolution & 11.4 pixel $/ 0.1 \mathrm{~mm}$ \\
\hline Welding speed & $2.5 \mathrm{~m} / \mathrm{min}$ & Welding materials & Austenitic stainless steel \\
\hline Shielding Gas(Ar) flow & $20 \mathrm{~L} / \mathrm{min}$ & $\begin{array}{l}\text { Specimen dimensions } \\
(\mathrm{L} \times \mathrm{W} \times \mathrm{D} \mathrm{mm})\end{array}$ & $150 \times 49 \times 10$ \\
\hline Camera frame rate & $1000 \mathrm{f} / \mathrm{s}$ & Seam gap & $0-0.1 \mathrm{~mm}$ \\
\hline
\end{tabular}

\section{Keyhole extraction}

One important step of using visual techniques to identify and track the welding seam is the extraction of the characteristic parameters of the keyhole. In the image of a workpiece, the gray level of the welds and the seam gap is not continuous. The seam gap is a straight line. Fig. 3 shows a molten pool infrared image, whose size is $512 \times 512$ pixel$^{2}$.

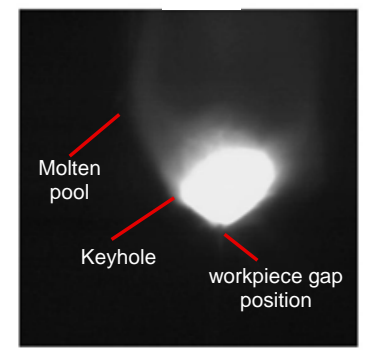

Fig.3 Molten pool infrared image

Fig.4 (a) also shows a typical low-resolution metal transfer image which captured with a frame rate 1000 frames/s. In the welding process, shown in Fig4(a), laser beam is focused on the workpiece to melt it and form the keyhole. Fig.4(a) shows an original keyhole image, the proposed method can be described in two steps.

Step1: Fixed threshold method was used to extract the keyhole of an image. The binary keyhole image was shown in Fig.4 (b).

Step2: The binary image was multiplied by the original gray image to obtain the keyhole gray image (Fig.4 (c)). 


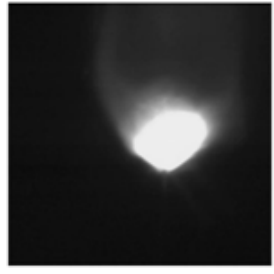

(a)

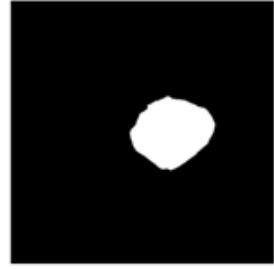

(b)

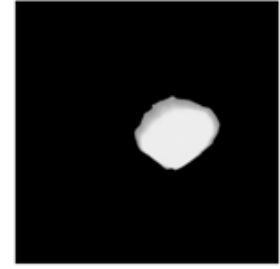

(c)

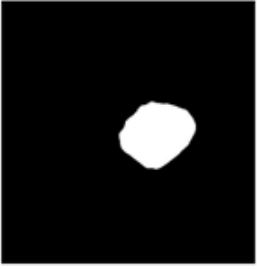

(d)

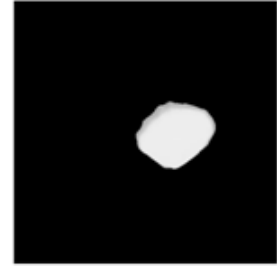

(e)

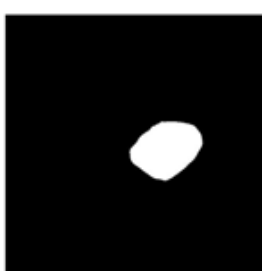

(f)

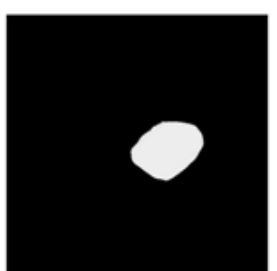

(g)

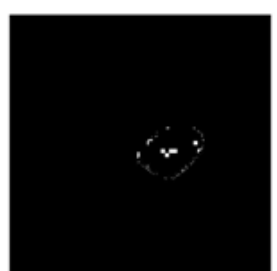

(h)

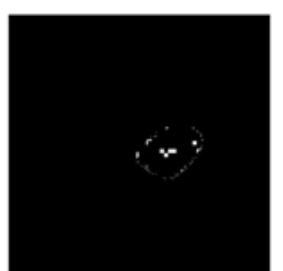

(i)

Fig.4 Processing of a keyhole image

(a) Original image (b) Binary image, threshold value is 0.55 (c) Keyhole image,threshold value is 0.55 (d)Binary image, threshold value is 0.65 (e) Keyhole image, threshold value is 0.65 (f) Binary image,threshold value is 0.85 (g) Keyhole image, threshold value is 0.85 (h) Binary image, threshold value is 0.93(i) Keyhole image, threshold value is 0.93

As shown in Fig4, with different thresholds, the size of the keyhole is inconsistent. Clearly, this will affect the value of the keyhole centroid.

\section{Keyhole centroid extraction}

The centroid of the keyhole image is the gravity of the image [4]. The calculation formula of the horizontal coordinate $(X)$ of the centroid of keyhole is shown in Eq.1.

$$
X=\frac{\sum_{i=1}^{M} \sum_{j=1}^{N} g(i, j) i}{\sum_{i=1}^{M} \sum_{j=1}^{N} g(i, j)}
$$

where $i$ is the abscissa value, $j$ is the value of the vertical ordinate. $M$ is the total row number, $N$ is the total column number and $g(i, j)$ is the pixel value of the point $(x, y)$.

In the welding process, tiny gap still exist between the two butt materials. Because heat conductivity is different in the air and welding materials, heat conduction will have greater "heat resistance" in the seam gap, and produce the heat accumulation effect [5]. Reflected in the infrared image, gray level of the weld will have a greater change. Similarly, the heat accumulation effect impact significantly on the keyhole centroid values.

When the laser beam is focused on the weld, the gray distribution of the keyhole image is symmetrical, the keyhole centroid should be aligned with the center line. If the gray distribution of the keyhole image is not symmetrical, the centroid of the keyhole will deviate from weld center line. As long as the quantitative relationship between the image centroid and the weld position established, the deviations of the laser beam and the weld will be calculated indirectly by the centroid of the keyhole. Fig.5 shows the horizontal coordinate values of the keyhole centroid with image sequences. It can be observed from the surface of welded specimen (Fig.2) that the laser beam gradually moved from one side of the weld to the weld center in the initial stage, the keyhole centroid also moves up from the below the center. In the middle stages of the welding process, the laser beam almost focused on the center line of the welds,. The keyhole centroid fluctuates around the center of the images. In the end of the welding process, the laser beam moved to theother side of the welds. For the same reason as initial stages, the keyhole centroid will move from center to the top the central value, seen from the experimental results,the keyhole centroid values has an increasing trend as the increase of weld deviations. 


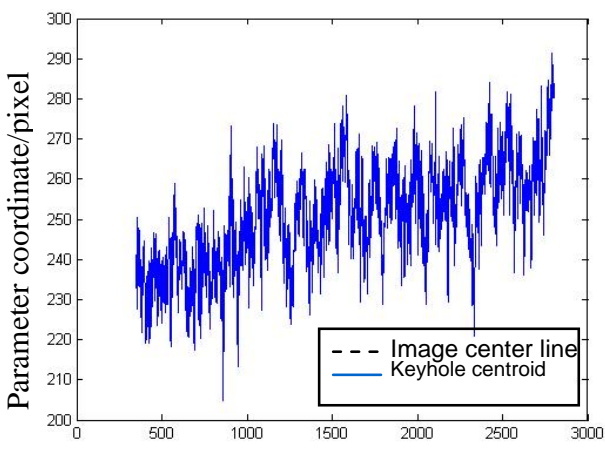

(a)

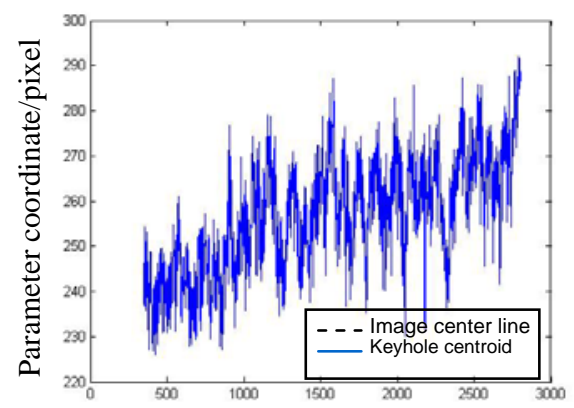

(c)

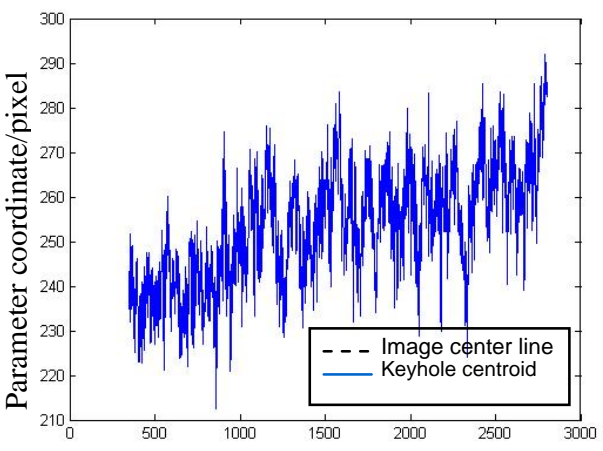

(b)

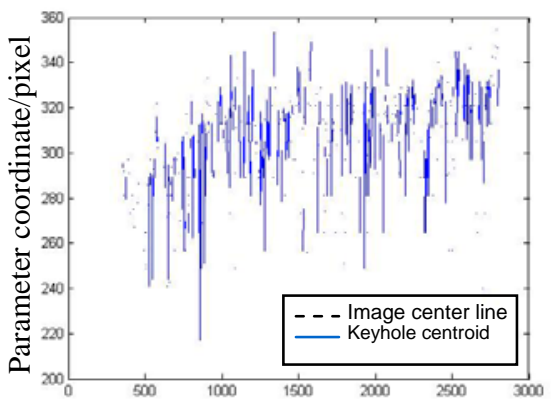

(d)

Fig.5 Horizontal coordinate values of the centroid of keyhole with image sequences,

(a) Samplings of keyhole images/f threshold value is 0.55, (b) Samplings of keyhole images/f, threshold value is 0.65, (c)Samplings of keyhole images/f, threshold value is 0.85, (d) Samplings of keyhole images/f, threshold value is 0.93

\section{Summary}

In the high-power fiber laser butt joint welding of Type 304 stainless steel, Extracted keyhole image from different threshold value $0.55,0.65,0.85$, and 0.93 , further, analyzed their differences. Relationship between the keyhole centroid and the welding seam was illustrated. The experimental showed the keyhole centroid values has an increasing trend as the increase of weld deviation.

\section{Acknowledgements}

This work was supported in part by the National Natural Science Foundation of China (51175095), the Guangdong Provincial Natural Science Foundation of China (10251009001000001, 9151009001000020) and the Specialized Research Fund for the Doctoral Program of Higher Education of China (20104420110001). Many thanks are given to Katayama Laboratory of Osaka University, for their assistance of laser welding experiments.

\section{References}

[1] Y.Kawahito, M.Kito and S.Katayama: J. Phys. D: Appl. Phys Vol 40, (2007), p.2972

[2] X.D.Gao, D.K.Ding,T.X.Bai and S.Katayama: IET Image Processing Vol 5, (2011), p.410

[3] X.D.Gao, L.Mo, X.G.Zhong, D.Y.You and S.Katayama: Acta Physica Sinica Vol 60, (2011), p.088105-1-8

[4] X.D.Gao and S. J. NA: Joumal Manufacturing Systems VOL 24,(2005), p.l

[5] A. P. Mackwood and R. C. Crafer: Laser Technol VOL 37, (2005) , p.99 